\title{
Face Detection Using Spectral Histograms and SVMs
}

\author{
Christopher A. Waring and Xiuwen Liu \\ Department of Computer Science, The Florida State University \\ Tallahassee, FL 32306 \\ chwaring@cs.fsu.edu liux@cs.fsu.edu
}

\begin{abstract}
We present a face detection method using spectral histograms and support vector machines. Each image window is represented by its spectral histogram, a feature vector consisting of histograms of filtered images. Using statistical sampling, we show systematically the representation groups face images together; in comparison, commonly used representations often do not exhibit this necessary and desirable property. By using a support vector machine trained on a set of 4500 face and 8000 non-face images, we obtain a robust classifying function for face and non-face patterns. With an effective illumination correction algorithm, our system reliably discriminates face and non-face patterns in images under different kinds of conditions. Our method on two commonly used data sets give the best performance among recent face detection ones. We attribute the high performance to the desirable properties of the spectral histogram representation and good generalization of support vector machines. Several further improvements in computation time and in performance are discussed.
\end{abstract}




\section{Introduction}

Face detection has gained increased interest in recent years. As computers become faster and more affordable, many applications that use face detection / localization are becoming an integral part of our life. For example, face recognition systems are being tested and installed at airports to provide a new level of security; human-computer interfaces based on facial expressions and body gestures are being exploited as ways to replace the traditional interfaces such as the mouse and the keyboard. These and other related applications all require as an initial step some form of face detection / localization, which can be simply defined as [32]: Given an image I, find all occurrences of faces and the extent of each face in I. This definition implies that some form of discrimination must be made between faces and all other objects. However, there are many difficulties and challenges associated with face detection. Variations in lighting conditions can make face images appear substantially different. Additives such as beards, mustaches, and glasses can augment the global structure of the face such as the jaw line and mask local features such as corners of the mouth. In addition, the large amount of intra-class variation ${ }^{1}$ amongst all faces makes reliable face detection intrinsically difficult.

At the core, face detection requires an effective discrimination function between face and non-face patterns. Accordingly, existing face detection approaches fall into one of four categories (see [32] for a recent survey): knowledge-based methods, template-based methods, feature invariant methods, or appearance-based methods. Knowledge-based methods

\footnotetext{
${ }^{1}$ While all faces posses to a certain degree a similar structure, location, alignment, size, and shape of facial features can vary greatly from person to person.
} 
(e.g. [9]) attempt to describe all the face patterns using rules based on human knowledge such as that all faces have two eyes and a mouth. However, they are difficult to use to detect faces in real images as the translation of human knowledge into well formed rules is nontrivial. If the rules are too restrictive, many faces will be ruled out, resulting in false negatives; on the other hand, if the rules are too general, non-face patterns will be included in the face class, resulting in false positives. Template-based methods (e.g. [35]) represent the face class by (a set of) templates with allowable deformations which rely on the alignment of feature points. However, feature points can become corrupted by lighting variations, pose, additives, or facial expression changes, making the alignment of feature nodes on the template to features on the input difficult. In addition, if the amount of deformations for templates are too constrained, faces may be missed during detection; in contrast, if the amount of deformations are too flexible, false detections will be introduced. Feature invariant methods (e.g. [34]) are hard to use in detecting faces in real images as it is difficult to find features that are truly invariant with respect to all faces and large perturbations in lighting, pose, and expressions. To overcome some of the difficulties, appearance-based methods (e.g. [21, 33, 27, 8, 28]) provide several key advantages and are widely used in face detection. Specifically, as they allow one to learn the models from training data, the large amount of intra-class variation, expression, and pose can be accounted for in training by using a large training set. However, as the training set is limited in practice and one is interested in detecting faces in images that have not seen, generalization becomes the key issue among appearance-based methods, which is largely determined by the underlying representation and the classifying function.

In this paper, we present an appearance-based method using spectral histograms [17] 
as representation and support vector machines [26] as classifier. Unlike some commonly used representations, the spectral histogram, shown through statistical sampling, gives good generalization by grouping only perceptually similar images together. With a support vector machine, this gives rise to a decision function that discriminates face and non-face patterns reliably in images under different kinds of conditions and results in best performance on two commonly used face datasets.

The rest of the paper is organized as follows. Section 2 introduces the spectral histogram representation and and shows its sufficiency for representing faces through sampling. Section 3 describes briefly support vector machines [26]. Section 4 discusses the proposed algorithm in detail, including the preprocessing stage, the training stage, the detection stage, and the post-processing stage. Section 5 shows our experimental results. Section 6 discusses possible further improvements in computation and performance. Section 7 concludes the paper.

\section{The Spectral Histogram Representation}

\subsection{Definition and Properties}

Given an input image $\mathbf{I}$ and a set of filters $\left\{F^{(\alpha)}, \alpha=1, \ldots, K\right\}$, a sub-band image $\mathbf{I}^{(\alpha)}$ is computed through linear convolution given by $\mathbf{I}^{(\alpha)}(v)=F^{(\alpha)} * \mathbf{I}^{(\alpha)}(v)=\sum_{u} F(u) \mathbf{I}(v-u)$. The histogram of each sub-band image $\mathbf{I}^{(\alpha)}$ is given by $\mathbf{H}_{\mathbf{I}^{(\alpha)}}(z)=\frac{1}{|\mathbf{I}|} \sum_{V} \delta\left(z-\mathbf{I}^{(\alpha)}(v)\right)$. The 


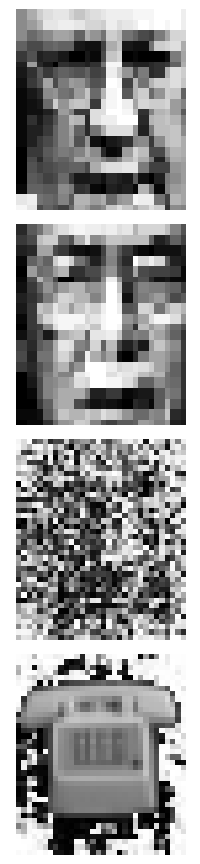

(a)
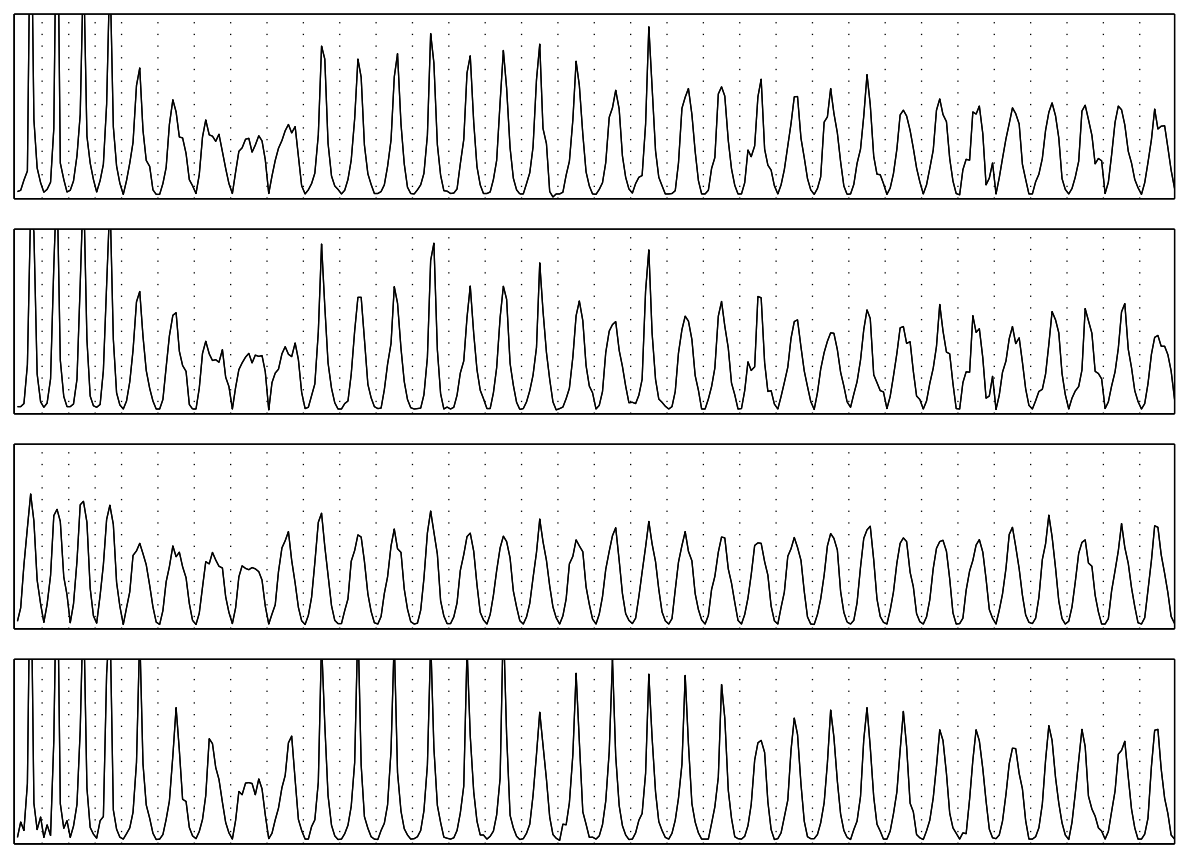

(b)

Figure 1: Spectral histogram of four images. (a) Input images. (b) The corresponding spectral histogram using the 33 chosen filters.

spectral histogram representation [17] of $\mathbf{I}$ with respect to the chosen filters is defined as the concatenation of $\mathbf{H}_{\mathbf{I}^{(\alpha)}}$, given by $\mathbf{H}_{\mathbf{I}}=\left(\mathbf{H}_{\mathbf{I}^{(1)}}, \ldots, \mathbf{H}_{\mathbf{I}^{(K)}}\right)$. In other words, each image is represented by the histograms of filtered images. Figure 1 shows the spectral histogram representation of several images using the 33 filters used throughout this paper (see below). These examples show that the spectral histograms of face images are similar while they are different for images from different classes.

In the spectral histogram representation, local features of an image are captured through filtering as the responses of individual filters depend on local structures and the global structures are implicitly captured by the constraints imposed by the histograms of different filtered images. The representation is non-parametric in nature and is effective to characterize dif- 
ferent kinds of patterns. One distinctive advantage of the spectral histogram representation is that two images do not need to be aligned in order to be compared (see Fig. 2 for examples) due to that the spectral histogram representation is not sensitive to perturbations of local image features. This reduces the number of required training images for both face and non-face classes.

To specify a spectral histogram representation, one needs to choose a set of filters. Following [37, 17], three different types of filters are used in this paper, including gradient filters, Laplacian of Gaussian filters, and Gabor filters. Gradient filters used include $D_{x}=\left[\begin{array}{lll}0.0 & -1.0 & 1.0\end{array}\right], D_{x x}=\left[\begin{array}{lll}-1.0 & 2.0 & -1.0\end{array}\right], D_{y}=\left[\begin{array}{lll}0.0 & -1.0 & 1.0\end{array}\right]^{T}$, and $D_{y y}=\left[\begin{array}{lll}-1.0 & 2.0 & -1.0\end{array}\right]^{T}$. Gabor Filters are defined as

$$
G(x, y \mid T, \theta)=e^{-\frac{1}{2 * T^{2}}\left(4(x \sin \theta+y \cos \theta)^{2}+(-x \cos \theta+y \sin \theta)^{2}\right)} e^{-i \frac{2 \pi}{T}(x \cos \theta+y \sin \theta)},
$$

where $T$ is the scale and $\theta$ the orientation. Laplacian of Gaussian Filters are given by $\operatorname{LoG}(x, y, T)=\left(x^{2}+y^{2}-T^{2}\right) e^{-\frac{x^{2}+y^{2}}{T^{2}}}$, where $T=\sqrt{2 \sigma}$ determines the filter's spatial scale, and $\sigma$ corresponds to the variance of the Gaussian distribution. The 33 filters used in this paper are:

- 4 Gradient filters $D_{x}, D_{y}, D_{x x}, D_{y y}$.

- 5 LoG filters with $\mathrm{T}=\sqrt{2} / 2,1,2, \sqrt{32} / 3($ expanded from $T=1)$ and $\sqrt{32} / 3($ expanded from $T=2$ ).

- 24 Gabor filters are used with $\mathrm{T}=2,5,12,16$ and $\Theta=0^{\circ}, 30^{\circ}, 60^{\circ}, 90^{\circ}, 120^{\circ}, 150^{\circ}$. 
To characterize face patterns more effectively, we have included large kernels to impose a more rigid global structure. By expanding smaller kernels to approximate the larger ones ${ }^{2}$, this is achieved without increasing computation. This set of filters captures the structures that are important to face patterns and is found to be effective for face detection in images under different kinds of conditions. Note, however, there is no optimality associated with the set. With a recent optimization technique proposed by Liu and Srivastava [15], an optimal set of filters for face detection can be learned by maximizing the performance on the training set, which needs to be further explored.

\subsection{Face Synthesis Through Sampling}

As discussed in the previous section, the underlying representation plays a critical role in generalization performance of any appearance-based face detection system. By analyzing generalization performance, Liu et al. [16] showed that a necessary condition for a good representation is that it should group only images from the same class together. For face detection, as we are concerned with the set of face patterns and the set of all other images, a good representation should group face patterns together and keep all other pattern groups away from the face ones. If the chosen representation groups patterns from different classes together, the detection performance from any classifier will be limited. If the chosen representation groups non-face patterns into the face class, there would be inherently false positive detections; likewise if the representation groups face patterns into the non-face class,

\footnotetext{
${ }^{2}$ This is implemented by $\mathbf{I}^{(\alpha)}(v)=\sum_{u} F(u) \mathbf{I}(v-k u)$; we have used $k=2, k=3$, and $k=4$.
} 
there will be inherently false negatives.

One way to analyze the intrinsic generalization of a given representation is to generate random samples from the set consisting of images with identical representations [16]. Technically, given a representation $\mathcal{G}$ and an image $\mathbf{I}_{\text {obs }}$ from a known class, we want to sample from $\left\{\mathbf{I} \mid \mathcal{G}(\mathbf{I})=\mathcal{G}\left(\mathbf{I}_{o b s}\right)\right\}$. Because of the high dimensionality of $\mathbf{I}$ (in this paper the dimension of $\mathbf{I}$ (a $21 \times 21$ window) is 441 ), enumeration is computationally infeasible. A common way to overcome this problem is to use statistical sampling techniques [30] and here we use a Gibber sampler with simulated annealing [36] on the induced Gibbs distribution given by

$$
q(\mathbf{I})=\frac{1}{Z_{\Theta}} \exp \left\{-\frac{\xi(\mathbf{I})}{\Theta}\right\}
$$

In (1), $\Theta$ denotes the temperature and $\xi(\mathbf{I})$ is called the energy, given by

$$
\xi(\mathbf{I})=D\left(\mathcal{G}(\mathbf{I}), \mathcal{G}\left(\mathbf{I}_{o b s}\right)\right)
$$

where $D$ is a distance measure between the representations. Intuitively, this is achieved by updating pixels according to the conditional probability computed from (1). For an algorithmic description of the sampling process, see Zhu et al. [36].

To demonstrate the sufficiency of the spectral histogram representation, we use it as $\mathcal{G}$ in (2), and use the following distance measure between two images,

$$
K L\left(\mathbf{H}_{\mathbf{I}_{1}}, \mathbf{H}_{\mathbf{I}_{2}}\right)=\sum_{\alpha=1}^{K} \sum_{z}\left(\mathbf{H}_{\mathbf{I}_{1}^{(\alpha)}}(z)-\mathbf{H}_{\mathbf{I}_{2}^{(\alpha)}}(z)\right) \log \frac{\mathbf{H}_{\mathbf{I}_{1}^{(\alpha)}}(z)}{\mathbf{H}_{\mathbf{I}_{2}^{(\alpha)}}(z)}
$$



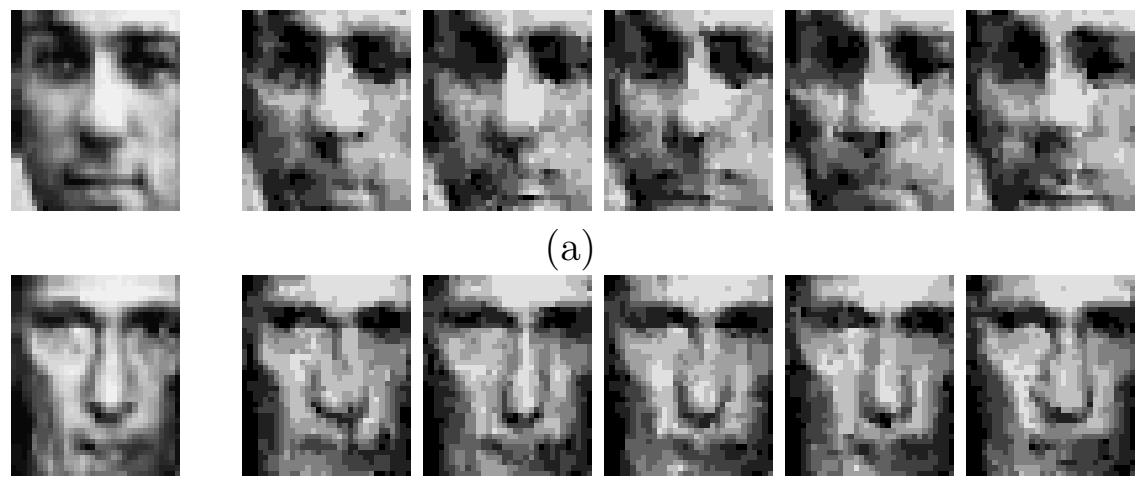

(b)

Figure 2: Two face images along with synthesized images through sampling using the spectral histogram representation. In each subfigure, the left most image is the original image and the rest are typical samples of the set with similar spectral histogram representation of the given one.

Figure 2 shows two examples of synthesized face images. In both cases, the spectral histogram representation captures the perceptual characteristics of the original images. These examples show that the spectral representation is able to capture important local features, e.g. the eyes, nose, and mouth. The global structure has also been captured as these features roughly lie in the locations where we would expect to see them in a face. Note that the variations amongst the synthesized face images are desirable as they show the spectral histogram representation groups perceptually similar patterns together.

In comparison, we have also analyzed the intrinsic generalization of linear representations $[2,12,19,31]$. We use the same Gibbs sampler except that the energy function is defined as the Euclidean distance between the projections of $\mathbf{I}$ and $\mathbf{I}_{o b s}$ along given linear basis. Figure 3 shows the results for the two images used in Fig. 2. In each subfigure, the bottom row shows the corresponding reconstructed image for the one given at the top. As expected, in each group, all the reconstructed images are identical as they all have the 
same linear representation. However, these images perceptually differ greatly and belong to different classes; this is because linear representations model pixels independently and thus allow some to change freely as long as their changes are cancelled by others. As these images have the identical representation, the use of a classifier cannot alleviate this problem. If a classifier classifies correctly the leftmost face images in Fig. 3, all other images must also be classified as face patterns, resulting in false positives. On the other hand, if a classifier classifies correctly the non-face images, the face images must be classified also as non-face patterns, resulting in false negatives. Because of this, methods based on linear representations are intrinsically sensitive to different patterns in the background.

It should be noted that reconstruction and sampling are very different. Sampling is concerned with the drawing of random samples from a set sharing the same representation while reconstruction is a deterministic procedure of recovering the original image from its low dimensional representation. To emphasize this point, Fig. 4 shows an illustration using a onedimensional linear representation. For the given image ' $x$ ', the corresponding reconstructed image is the point ' + ' shown in Fig. 4(a) while the sampling can give any point along the solid line shown in Fig. 4(b). This shows an important point that the sufficiency of a representation cannot be solely justified based on the reconstruction accuracy; statistical sampling techniques must be used in order to analyze its generalization properties. 

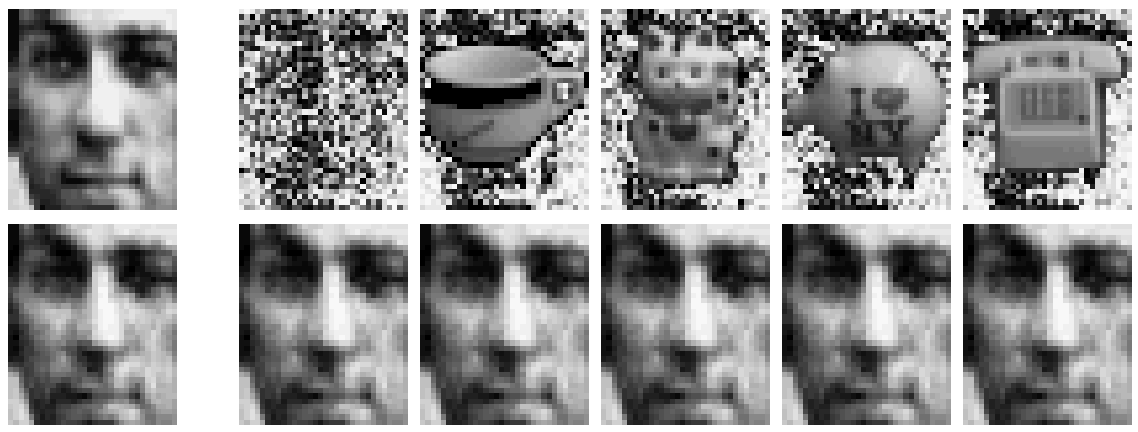

(a)
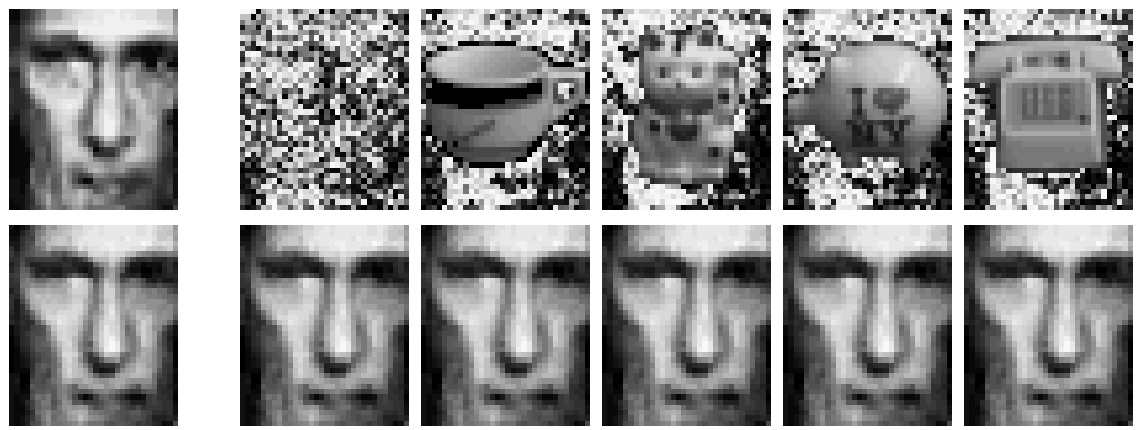

(b)

Figure 3: Synthesized images of faces using linear representations. Here the basis is generated using PCA (principal component analysis). In each subfigure, the top row shows the original image (the leftmost one) and five typical samples from the set with the same linear representation; the bottom row shows the corresponding reconstructed image to show the fact that all of the images indeed have the same linear representation. Note that in the four synthesized images on the right, the center object is used as the boundary condition during sampling in that they are not updated.

\section{Support Vector Machines}

In this paper we choose the support vector machine $[26,20]$ as the classifying function.

One distinctive advantage this type of classifiers has over traditional neural networks is that support vector machines achieve better generalization performance. While neural networks such as multiple layer perceptrons (MLPs) can produce low error rate on training data, there is no guarantee that this will translate into good performance on test data. Multiple layer perceptrons minimize the mean squared error over the training data (empirical risk 


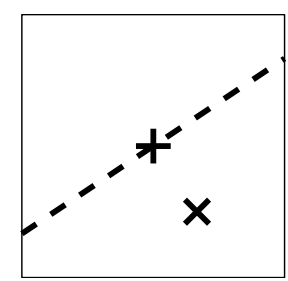

(a)

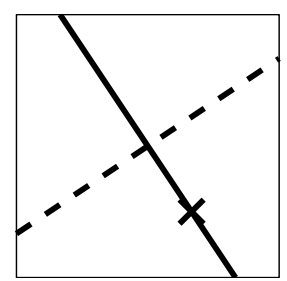

(b)

Figure 4: Illustration of the difference between sampling and reconstruction. Here the dashed line represents a one-dimensional subspace in a two-dimensional space. For a given (face) image (marked as ' $\mathrm{x}$ '), the sampling is to draw a random point along the solid line in (b) while the reconstructed image is a single point given by ' + ' in (a).

minimization) where support vector machines use an additional principal called structural risk minimization [26]. The purpose of structural risk minimization is to give an upper bound on the expected generalization error.

To illustrate the difference, Fig. 5 shows a toy example with two classes. While there are numerous such decision planes that can give zero error on the training set (three are shown in Fig. 5(a)), the performance of these decision boundaries will vary when applied to unseen data. Fig. 5(b) shows the decision boundary given by the support vector machine, constructed as the one that separates two classes with the maximum distance between their margins. As shown by Vapnik [26], this maximal margin decision boundary can achieve optimal worst case generalization performance. Note that support vector machines are originally designed to solve problems where data can be separated by a linear decision boundary. By using kernel functions (see Osuna et al. [20] for details), SVMs can be used effectively to deal with problems that are not linearly separable in the original space. Some of the commonly used kernels include Gaussian RBF (Radial Basis Functions), polynomial functions, and sigmoid polynomials. In this paper we choose the Gaussian RBF kernel function on 


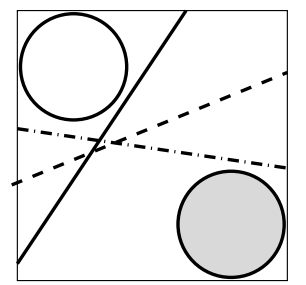

(a)

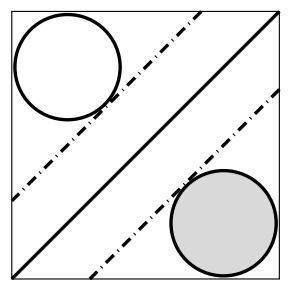

(b)

Figure 5: A toy example that shows a small set of possible decision boundaries for an MLP and the decision boundary for an SVM. (a) A set of possible decision boundaries that could be derived by an MLP. Each line depicts a possible decision boundary. (b) The decision boundary given by an SVM. The thick line is the decision boundary and the two dotted lines are the margins for the decision boundary.

the spectral histogram representation of the input ${ }^{3}$. As the spectral histogram is naturally normalized, it simplifies the choice of parameters for the kernel function and the SVM. Our implementation of SVMs is based on the SVM-light package by Thorsten Joachims ${ }^{4}$.

\section{Face Detection}

This section discusses in detail each stage of the proposed method including preprocessing, training, detection, and post-processing.

\footnotetext{
${ }^{3}$ In a previous study [28], we used an MLP as the classifier and obtained slightly worse results; within SVMs, one can use different kernel functions and we expect the results would be similar.

${ }^{4}$ Obtained from http://svmlight.joachims.org.
} 


\subsection{Preprocessing}

As nonuniform illumination can alter the appearance of features in images, it is imperative to correct this effect. We use a modified version of a method proposed by Sung and Poggio [25]. For each $7 \times 7$ block, the minimum value is computed, which generates a $3 \times 3$ minimal brightness plane on a $21 \times 21$ window. The brightness plane is then resized by bilinear interpolation to a $21 \times 21$ minimal brightness plane. The resulting minimal brightness planes for the images shown in Fig. 6(a) are given correspondingly in Fig. 6(b). The minimal brightness plane is then subtracted from the original image. As shown in Fig. 6(c), the procedure corrects for the effect of non-uniform illumination on the original images. Finally, the illumination corrected images are further normalized by applying histogram equalization and the preprocessed images are shown in Fig. 6(d). These preprocessing steps are applied to all the face and non-face training images and each window in each test image. Compared to Sung and Poggio's original iterative gradient method [25], our algorithm is computationally simpler and seems sufficient for detection using the spectral histogram representation.

\subsection{Training}

The training face images ${ }^{5}$ were generated from real images (separate from the images used in test) by fitting a $21 \times 21$ image window around each face using an affine transform based on eye positions as described in [25]. The original set consists of 1500 images and here we increased

\footnotetext{
${ }^{5}$ These training images, along with the test ones were provided by Henry Schneiderman from Carnegie Mellon University and were used in [21, 25, 22].
} 

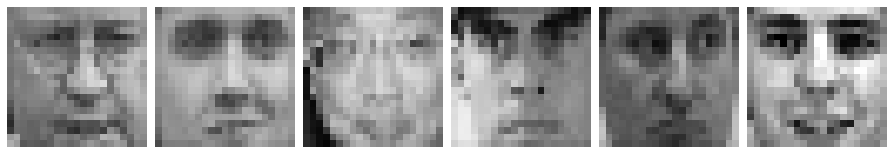

(a)
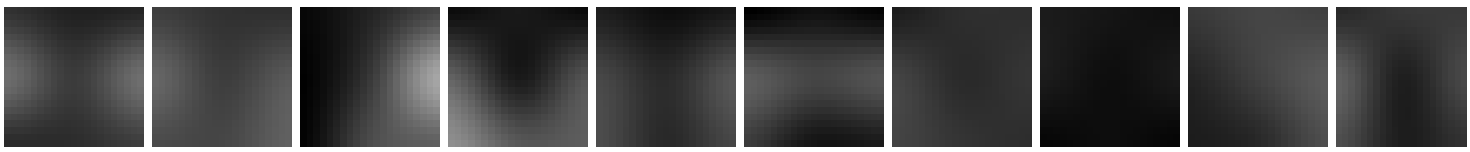

(b)
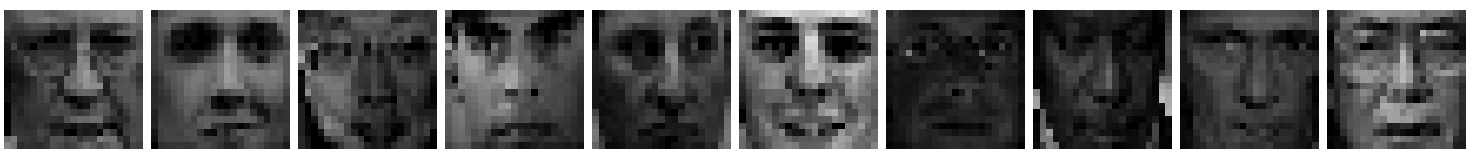

(c)
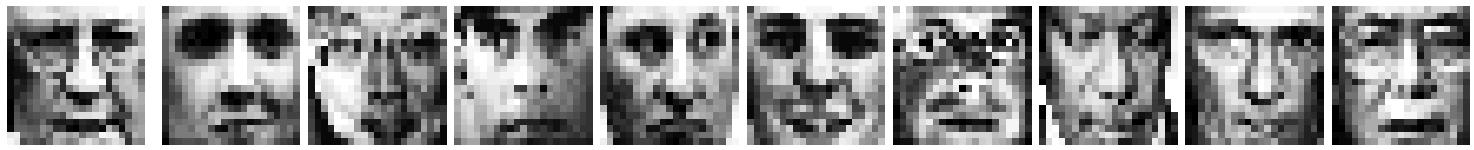

(d)

Figure 6: Steps in the preprocessing stage. (a) Ten $21 \times 21$ images from the training set. (b) The corresponding minimal brightness plane for each face image. (c) Illumination corrected images by subtracting the minimal brightness plane from the original image. (d) Preprocessed images.

it to 4500 by rotating each image randomly by $\mathrm{r}$ and $\mathrm{r}$ where $\mathrm{r}$ ranges from $0^{\circ}<r \leq 15^{\circ}$. This increases the rotation invariance of the proposed method. A problem particular to face detection is how to select effective non-face training images. As non-face images include all kinds of images, a prohibitively large set is needed in order to be representative, which would also require infeasible amount of computation in training. To alleviate this problem, a bootstrapping method, proposed by Sung and Poggio [25], is used to incrementally build the non-face images in the training set. Starting with 1500 random non-face images, we generated in total 8000 non-face images using the bootstrapping method.

All the training images, 4500 face and 8000 non-face ones, are first preprocessed according 


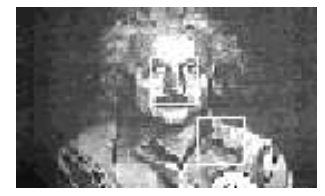

(a)

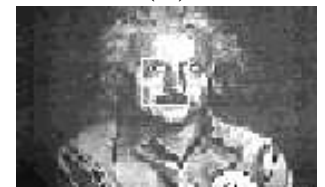

(e)

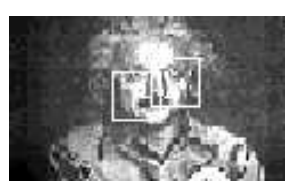

(b)

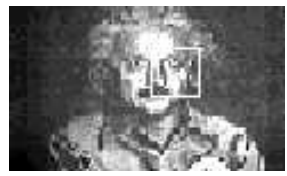

(f)

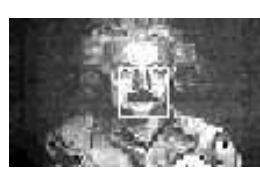

(c)

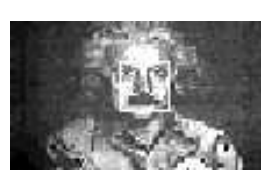

(g)

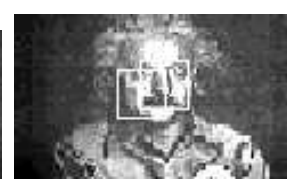

(d)

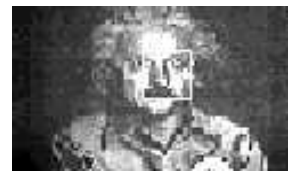

(h)

Figure 7: An example that illustrates the difference between no thresholding and adaptive thresholding. Images in (a)-(c) were created with no thresholding used on the region growing results and (d) shows the final result. Images in (e)-(g) were created using an adaptive threshold of $2 / 3$ and (h) shows the final result.

to the preprocessing stage discussed previously. The spectral histogram of the illumination corrected face and non-face images in the training set is then calculated using the 33 filters. The resulting spectral histograms are used as input to train a support vector machine with a RBF kernel function. The trained SVM is then used to classify image windows in test images.

\subsection{Detection and Post-processing}

As the discrimination function is designed on a $21 \times 21$ image window, it implicitly requires that all faces lie roughly within a $21 \times 21$ window. Here a three level Gaussian pyramid is built by successively down sampling the test image by a factor of 1.1. Each level of the pyramid is processed independently at the detection stage. This achieves some degree of scale invariance. For the image at each level, a $21 \times 21$ image window is moved pixel by pixel canvassing the entire image. Each test window is normalized using the method outlined in Sec. 4.1. The spectral histogram is then computed using the 33 chosen filters and stored. 
Once all the histograms have been computed, the histogram matrix is fed to the trained SVM for classification. If a local window is classified as a face, its center is saved. A region growing algorithm is used to coalesce nearby positive outputs (given by the SVM) in small regions and thresholding on the number of positive outputs is then applied to each region.

An important question is how to choose a proper threshold to achieve good performance on different kinds of images. For instance, if an arbitrary threshold is set, the generalized performance may not be good. One can test a set of images and find some average threshold [21]. However the number of positive outputs at a face region can vary from image to image. Therefore, a threshold that changes with images is desirable. Using a set of 20 images ranging from single face images to images with multiple faces, we found empirically that in all the images face regions possess the highest number of positive outputs per region. The number of positive outputs per region for each face $\alpha_{f_{a c e}}$ was found to fall within $\frac{\alpha_{\max }}{2}<\alpha_{\text {face }_{i}} \leq \alpha_{\max }$, where $\alpha_{\max }$ denotes the maximum positive outputs per region for an image. Instead of using a fixed value, the threshold of positive outputs per region is given by $\delta \times \alpha_{\max }$; as $\alpha_{\max }$ can vary from image to imagei, thus the threshold. We call this adaptive thresholding. Three different values, with respect to $\alpha_{\max }, \delta=\frac{1}{2}, \frac{2}{3}$, and $\frac{3}{4}$, were tested. $\delta=\frac{2}{3}$ was found to give the best overall performance. With $\delta=\frac{1}{2}$ the majority of faces were detected, but there were many false detections. Increasing $\delta$ from $\frac{1}{2}$ to $\frac{2}{3}$ only slightly decreased the number of face detections, but sharply curbed the number of false detections. When $\delta$ was increased from $\frac{2}{3}$ to $\frac{3}{4}$, the number of faces detected and false detections dropped substantially. Figure 7 shows the difference between no thresholding and adaptive thresholding. Notice that when no threshold is applied as in Fig. 7(d), there are 


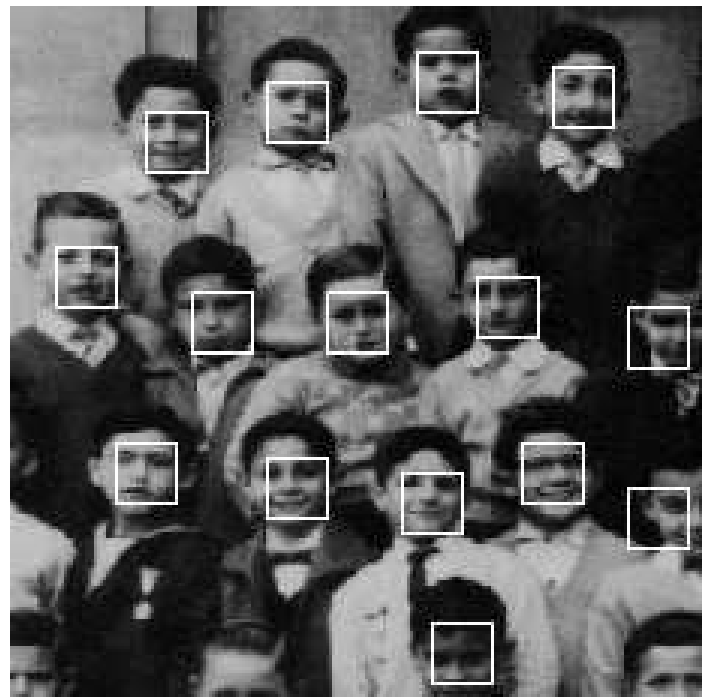

(a) $15 / 15 / 0$
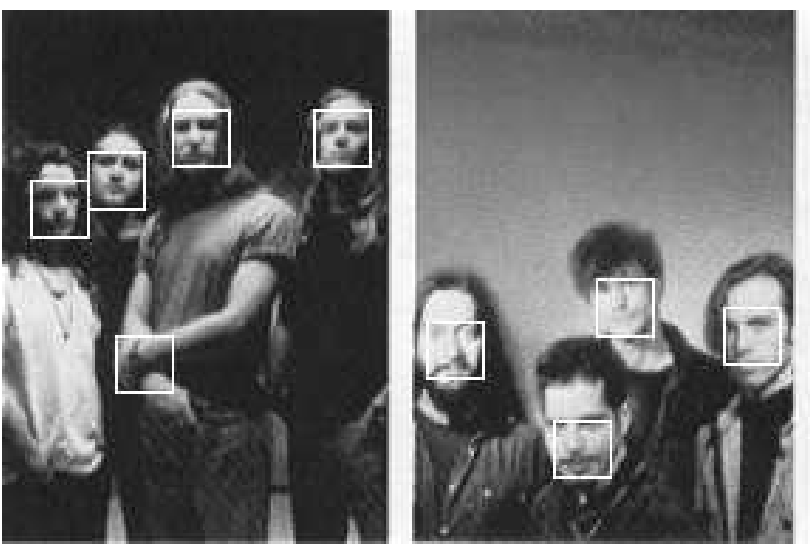

(b) $8 / 8 / 1$

Figure 8: Some typical results on test set 1. In each panel, the first number is the total number of faces in the image (the given ground truth), the second one the number of faces detected by the proposed algorithm, and the third one the number of false detections given by the proposed algorithm.

two final detections over the face. In contrast when adaptive thresholding is utilized, there is only one final detection as shown in Fig. 7(h).

Instituting adaptive threshold $\delta=\frac{2}{3}$, any region with fewer positive outputs from SVM than $\delta \times \alpha_{\max }$ is discounted as a non-face. After region growing and thresholding, regions that are less than three pixels apart are coalesced. The centroid for each new region is computed and saved. Once the entire image pyramid has been processed, detections at each layer are examined. A detection is marked as final if and only if it is found in at least two concurrent levels of the image pyramid. A similar hierarchal approach was used by Rowley et al. [21] and Juell et al. [8]. A detection is registered as correct if it contains half or more of a face. Otherwise it is labeled a false detection. 


\section{$5 \quad$ Experimental Results}

The proposed face detection algorithm was applied to two commonly used data sets that are separate from the training images. Test set 1 consists of 125 images containing 483 faces and test set 2 consists of 23 images containing 136 faces. Test set 1 was originally used by Rowley et al. [21] and has become one of the standard test sets for face detection algorithms. Test set 2 was initially used by Sung and Poggio [25]. Figures 8 - 11 show some of the typical results on test set 1 from the proposed algorithm. As is evident from these results our algorithm demonstrates exceptional performance on images that range from high quality to low quality. Figure 8 shows two examples with relative simple background and the proposed algorithm detects all the faces accurately even when some of them are rotated (Fig. 8 (b)). Figure 9 shows three examples with perfect performance. In Fig. 9(c), the man's face is detected correctly despite that his eyes are occluded by the visor that he wears and the complex background. In Fig. 10 we detect all instances of faces with one false detection located on the womans hands on the top level. In Fig. 11 we are able to detect all 57 faces with no false detections. Note that this image contains many different patterns and faces are close to one another, making detection in this type of images difficult. Our algorithm not only detects all the instances of faces, but also marks the detections accurately.

Figures 12-14 show typical results from the proposed algorithm on test set 2. In Fig. 12, all the faces are detected correctly and accurately. Figures 13 and 14 depict two low quality images. In Fig. 13 there is a variety of expressions on the faces and each face is posed differently. In Fig. 14, notice the three larger faces in the bottom right corner. These faces 


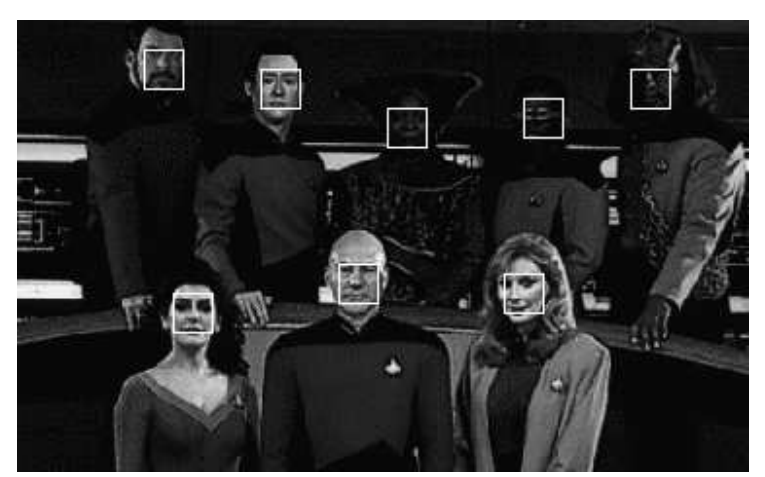

(a) $8 / 8 / 0$

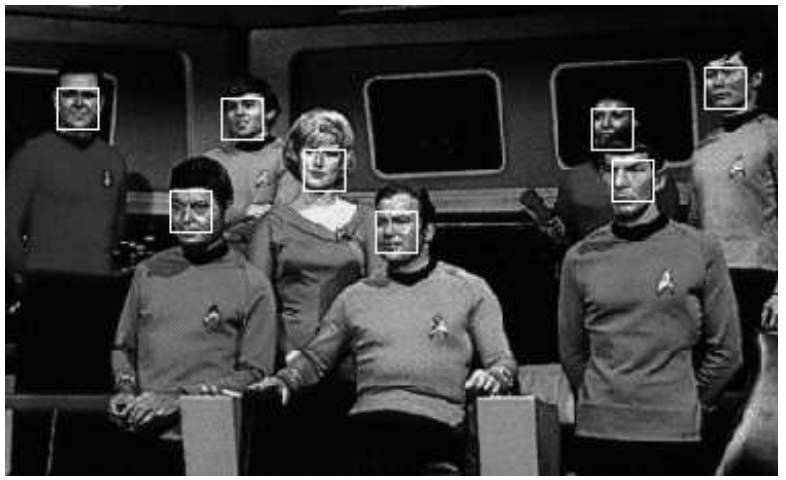

(b) $8 / 8 / 0$

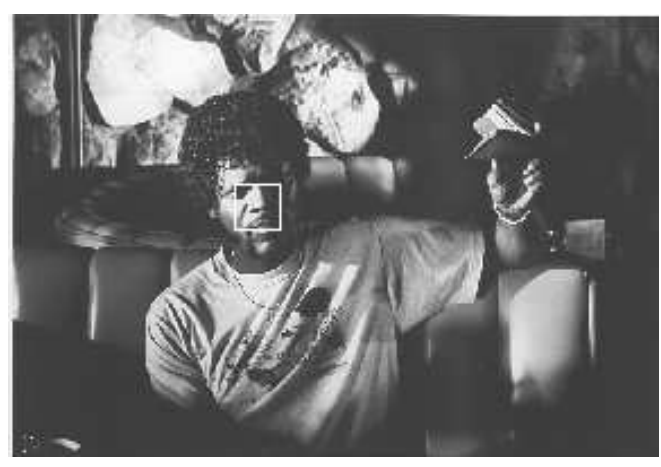

(c) $1 / 1 / 0$

Figure 9: Some more typical results on test set 1. See Fig. 8 for the legend.

were too large to fit entirely in the $21 \times 21$ image window, but we are still able to detect them. As a result, our algorithm detects all the faces despite the low quality of images in Fig. 13 and 14 .

While there are still false detections given by the proposed algorithm, unlike many other methods, the false detections in our results are localized in very specific areas and thus could be handled effectively. The majority of false detections are localized around the hands such as in Fig. 8(b) and Fig. 12(c). This can be attributed to the relaxed topological ordering imposed by the spectral histogram representation. One can overcome this problem by either including images of hands in certain positions as negative training examples, or imposing a stricter topological structure. The latter can be accomplished by computing the spectral 


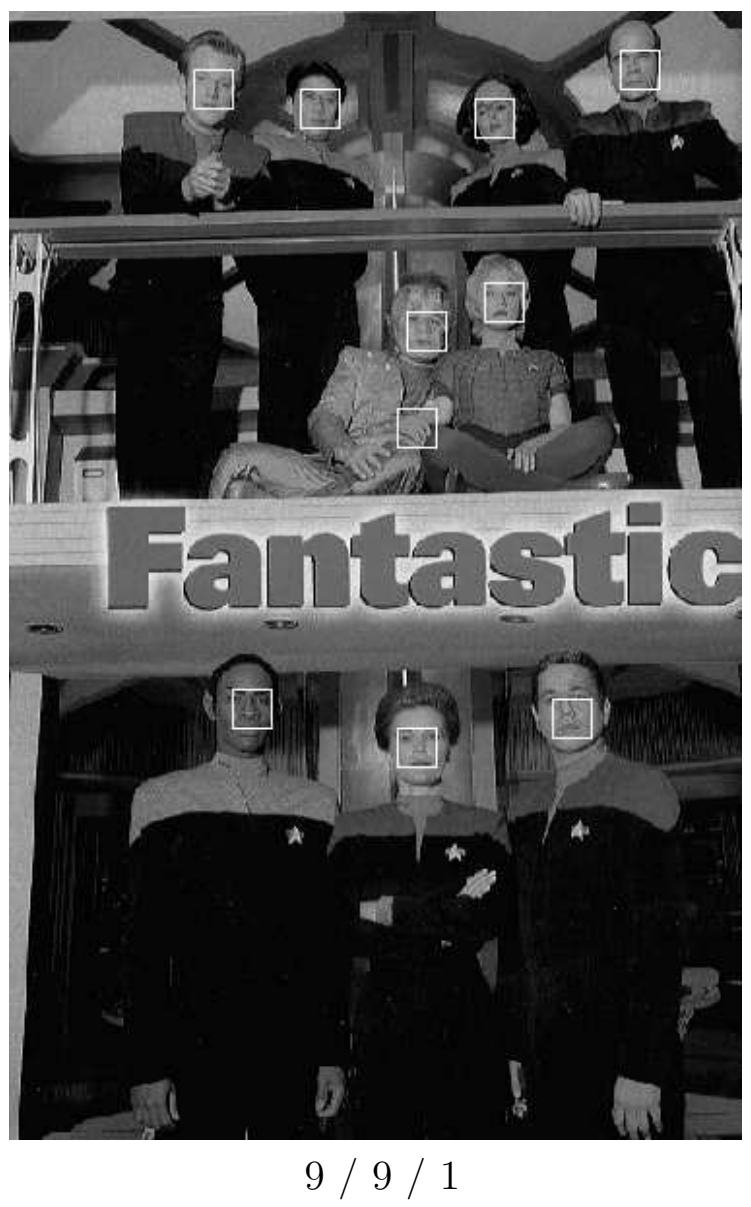

Figure 10: Some typical results on test set 1. See Fig. 8 for the legend.

histograms for specified subsections of an image window [13]. This would enforce a more rigid alignment of feature points as there would be less variation within each sub-region of the window compared to the total variation within the entire window.

Table 1 shows a summary of our results along with that from other recent methods on the same test sets. The results from other methods are taken from Yang et al. [32]. As is evident from these results, our algorithm gives the best overall performance. 


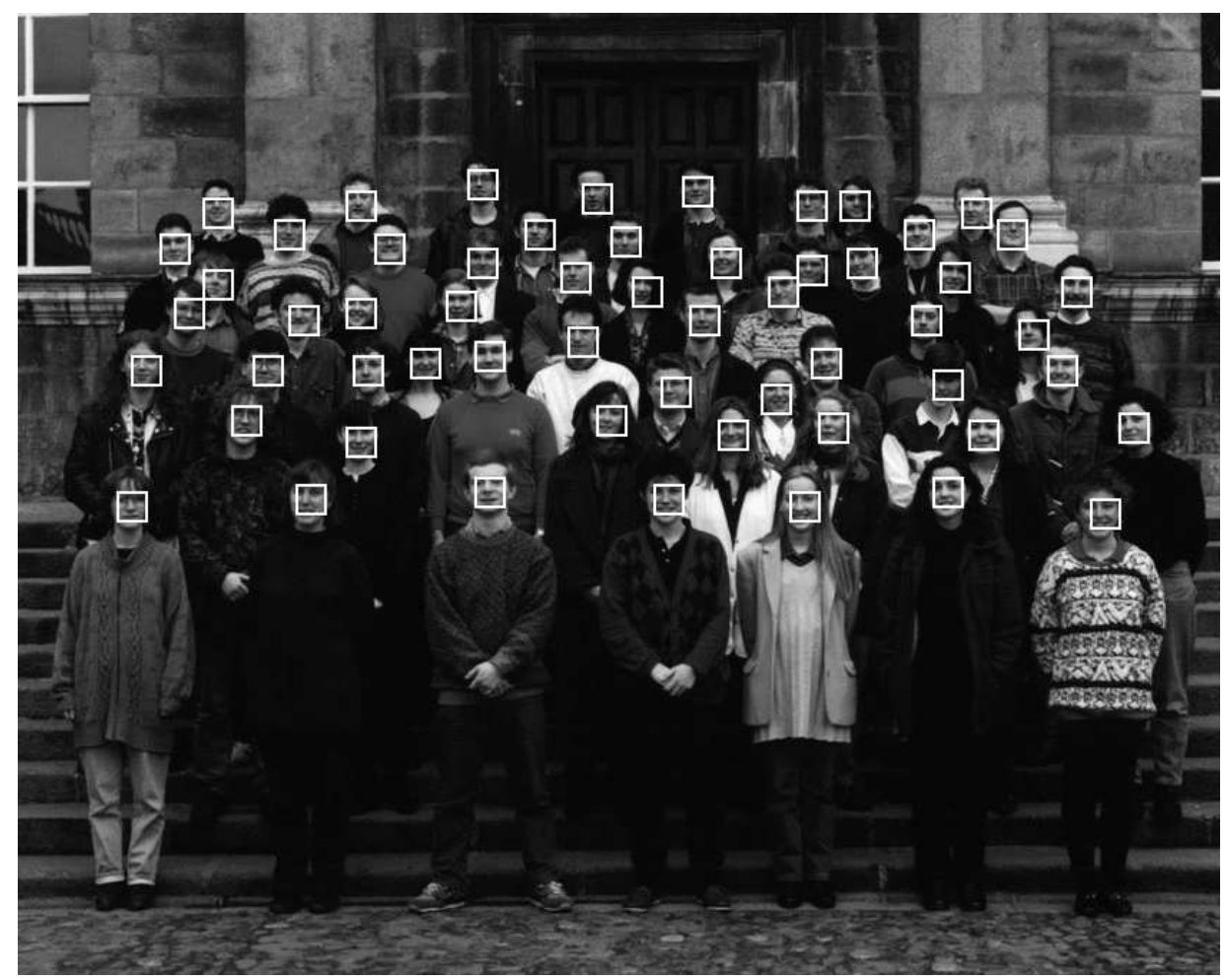

$57 / 57 / 0$

Figure 11: Some more typical results on test set 1. See Fig. 8 for the legend.

\section{Discussion}

We have presented a face detection method based on spectral histogram representations and support vector machines and have demonstrated its performance on two commonly used data sets. The comparison shows that our method achieves the best performance with respect to both false detections and detection rate. The performance of our system can be attributed to the desirable properties of spectral histogram representations and the generalization property of support vector machines. One may further improve the performance by integrating the spectral representation with other representations with more rigid topological ordering such as edge maps [5], Gabor jets [10], or templates [35] and thus reducing further the number of false positives. 


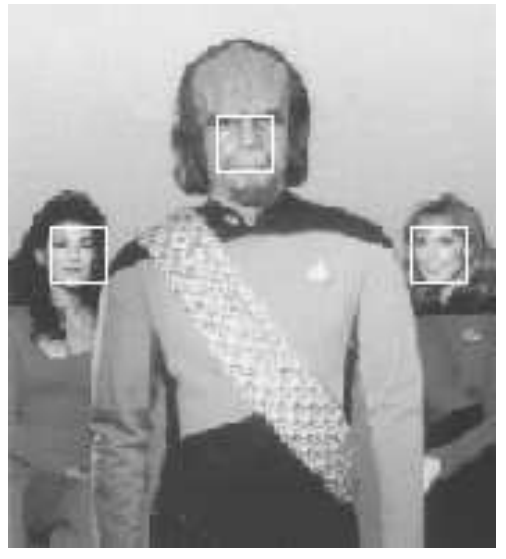

(a) $3 / 3 / 0$

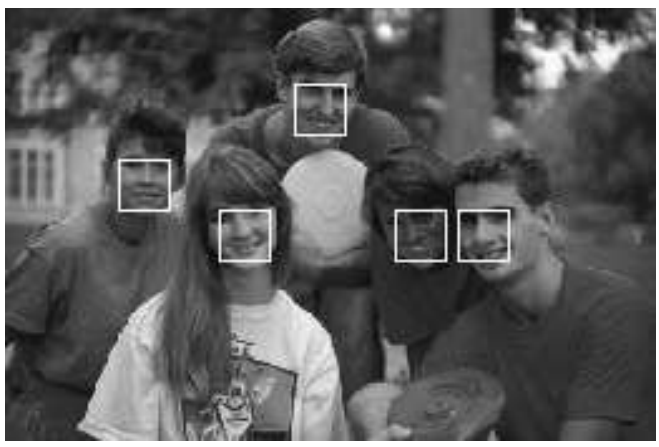

(b) $5 / 5 / 0$

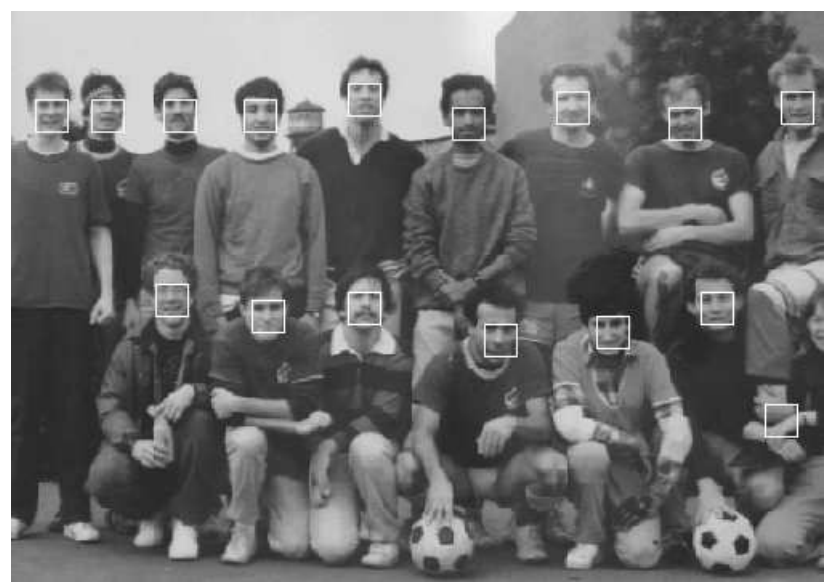

(c) $15 / 15 / 1$

Figure 12: Some more typical results on Test set 2. See Fig. 8 for legend.

In addition to the performance, this type of representations offers further benefits. For example, our approach is generic in nature and is applicable to other forms of object detection and recognition, not solely face detection. As shown by Liu et al. [16, 15, 14], the spectral representation can be used to enhance the performance on such tasks as face and object recognition. This could lead to the possibility of integrating the two systems as one. While several other methods (e.g. $[22,27])$ are applicable to detection of different objects, none of them has shown to be effective for recognition and classification of faces, objects, and textures. Due to the filters used, the proposed system exhibits a large degree of rotation 


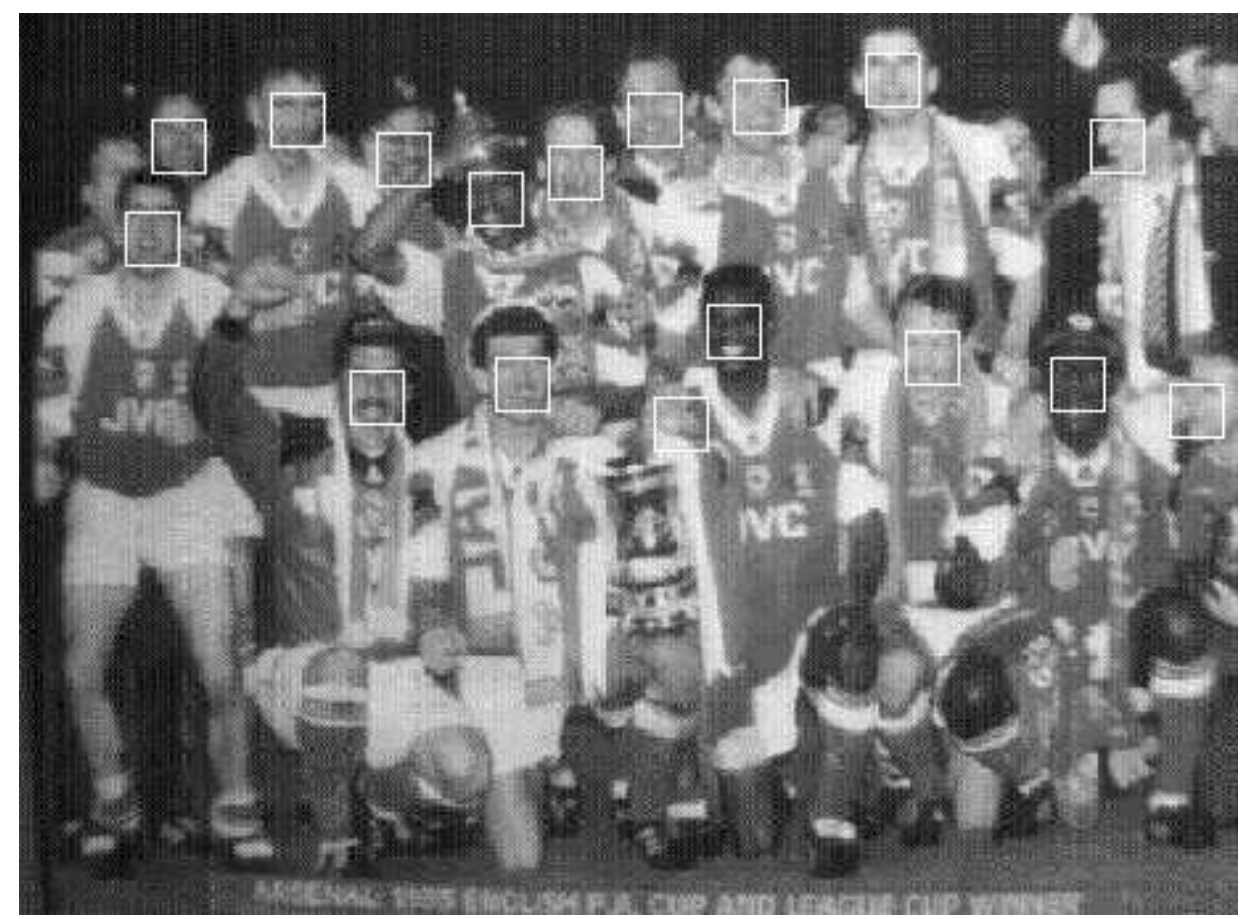

$16 / 16 / 1$

Figure 13: Some more typical results on Test set 2. See Fig. 8

invariance, which is demonstrated in Fig. 15. Results in Fig. 15 (a) and (b) are both generated with no modification to the current system as they are tested using the same procedure for all images in the test sets. Note the faces with large rotation are detected correctly. By combing the results in (a) and (b), all the faces are detected correctly with no false detections as shown in Fig. 15 (c). This example demonstrates that our system can handle rotation up to $45^{\circ}$.

As the spectral histogram representation has been applied to recognition in different modalities such as infrared images [23, 14], it may also provide an effective representation to detect faces in infrared and other kinds of images. As shown by Srivastava and Liu [23], infrared imaging is not sensitive to illumination conditions and thus face detection based on infrared images may operate during day and night times. A context-sensitive face detection method may also be possible through the use of spectral histograms based on their 


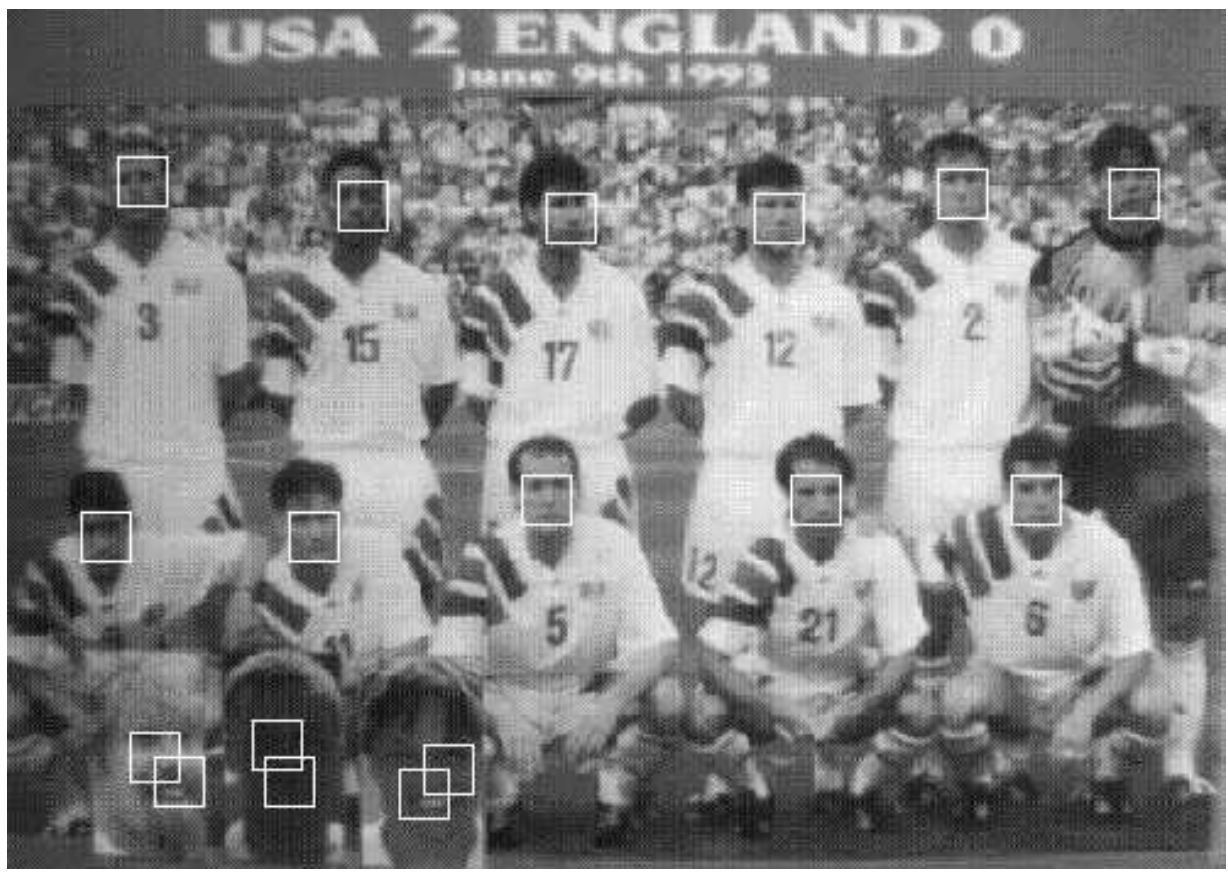

$14 / 14 / 0$

Figure 14: Some more typical results on Test set 2. See Fig. 8

effectiveness in clutter modeling [7, 24].

Compared to some recent methods (e.g. [27, 11]), our method is significantly slower. It takes several minutes to process a typical test image. While spectral histograms of local windows can be calculated efficiently using integral histogram images [13], it takes time for the SVM to classify these large number of local windows. There are a few possible ways to reduce the computation time. One possibility is to use Adaboost-like algorithms $[27,11]$ to learn much more efficient classifiers. As these computations are intrinsically parallel, another way is to implement them on parallel machines or special-purpose chips such as FPGAs [29]. There are some other possible ways to decrease the running time. The image window could be moved every two or three pixels instead of every pixel. This would certainly lead to faster run times, however the performance may suffer as the number of detections per region for 
Table 1: Results from our method along with that from others.

\begin{tabular}{|l|c|c|c|c|}
\hline & \multicolumn{2}{|c|}{ Test set 1 } & \multicolumn{2}{c|}{ Test set 2 } \\
\hline Method & $\begin{array}{c}\text { Detection } \\
\text { Rate }\end{array}$ & $\begin{array}{c}\text { False } \\
\text { Detections }\end{array}$ & $\begin{array}{c}\text { Detection } \\
\text { Rate }\end{array}$ & $\begin{array}{c}\text { False } \\
\text { Detections }\end{array}$ \\
\hline Waring \& Liu & $96.7 \%$ & 67 & $95.6 \%$ & 6 \\
\hline Yang, Ahuja, \& Kriegman [31] & $93.6 \%$ & 74 & $91.5 \%$ & 1 \\
\hline Yang, Ahuja, \& Kriegman [31] & $92.3 \%$ & 82 & $89.4 \%$ & 3 \\
\hline Yang, Roth, \& Ahuja [33] & $94.2 \%$ & 84 & $93.6 \%$ & 3 \\
\hline Yang, Roth \& Ahuja [33] & $94.8 \%$ & 78 & $94.1 \%$ & 3 \\
\hline Rowley, Baluja, \& Kanade [21] & $92.5 \%$ & 862 & $90.3 \%$ & 42 \\
\hline Schneiderman [22] & $93.0 \%$ & 88 & $91.2 \%$ & 12 \\
\hline Colmenarez \& Huang [3] & $98.0 \%$ & 12758 & N.A. & N.A \\
\hline Sung \& Poggio [25] & N.A. & N.A. & $81.9 \%$ & 13 \\
\hline
\end{tabular}

faces may decrease. This can be further improved by moving the windows adaptively. In regions with low scores from the trained SVM, we can move more pixels; in regions with high scores we can move detection windows pixel by pixel. This would reduce dramatically the computation and yet not reduce the number of detections in face regions. Another way is to have several face detectors that are organized hierarchically based on their computation complexity as in $[1,5]$. A possible way to implement such a hierarchy for face detection is to use spectral histograms with different number of filters. With fewer filters, one can obtain candidate regions faster and then apply more accurate spectral histogram models only on those candidate regions. This needs to be further explored.

\section{Conclusion}

In this paper we have shown that the spectral histogram representation is a good choice for face detection and yields results that are better than other recent methods both with respect 


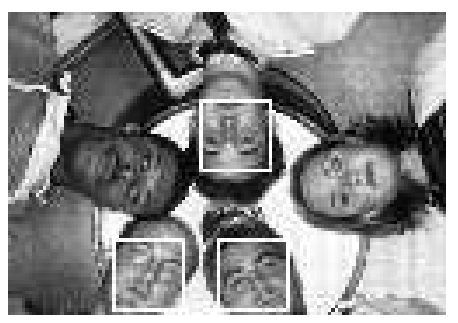

(a) $5 / 3 / 0$

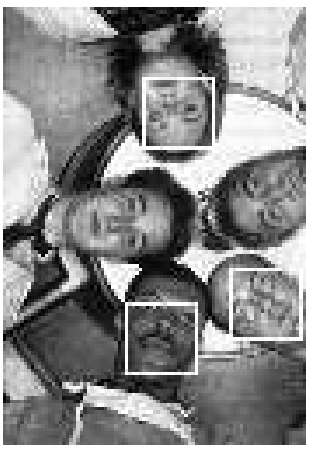

(b) $5 / 3 / 0$

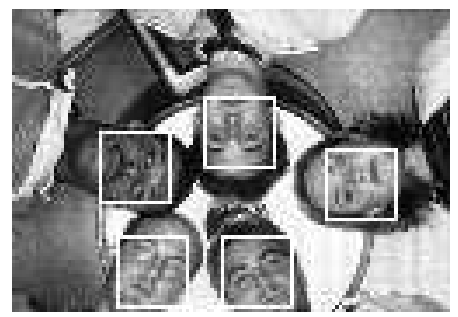

(c) $5 / 5 / 0$

Figure 15: An example that demonstrates large rotation invariance of the proposed method. (a) and (b) Results generated with the current system without modification. Note that the image in (b) is obtained by rotating that in (a) for $90^{\circ}$. (c) Results by combining the results from (a) and (b). See Fig. 8 for the legend.

to false detections and detection rate. In addition, without any modification our system is able to achieve a respectable degree of rotation invariance. While most representations used in face detection are justified only based on empirical results, the sufficiency of the spectral histogram representation is shown systematically through statistical sampling. As our representation is generic in nature, it can be easily adapted to other forms of object detection and other tasks such as face recognition [16], object recognition [16], and texture classification [18]. With these results, we expect the spectral histogram representation may provide a unified representation for effective object detection and recognition.

\section{Acknowledgments}

The authors would like to thank the anonymous reviewers whose comments have improved the presentation of this paper significantly. This research was supported in part by NSF IIS-0307998 and NGA NMA 201-01-2010. 


\section{References}

[1] Y. Amit and D. Geman, "A computational model for visual selection," Neural Computation, vol. 11, pp. 1691-1715, 1999.

[2] P. Belhumeur, J. Hespanha, and D. Kriegman, "Eigenfaces vs. fisherfaces: recognition using class specific linear projection," IEEE Transactions on Pattern Analysis and Machine Intelligence, vol. 19, no. 7, pp. 711-720, 1997.

[3] A. Colmenarez and T. Huang, "Face detection with information-based maximum discrimination," in Proceedings of the IEEE Conference on Computer Vision and Pattern Recognition, 1997, pp. 782-787.

[4] J. Daugman, "Uncertainty relation for resolution in space, spatial frequency, and orientation optimized by the two-dimensional visual cortical filters," Journal of the Optical Society of America, A, vol. 2, no. 7, pp. 23-26, 1985.

[5] F. Fleuret and D. Geman, "Coarse-to-fine face detection," International Journal of Computer Vision, vol. 41, pp. 85-107, 2001.

[6] S. Geman, E. Bienenstock, and R. Doursat, "Neural networks and the bias/variance dilemma," Neural Computation, vol. 4, pp. 1-58, 1992.

[7] U. Grenander and A. Srivastava, "Probability models for clutter in natural images," IEEE Transactions on Pattern Analysis and Machine Intelligence, vol. 23, no. 4, pp. 424-429, 2001. 
[8] R. Juell and R. Marsh, "A hierarchal neural network for human face detection," Pattern Recognition, vol. 29, pp. 781-787, 1996.

[9] C. Kotropoulos and I. Pitas, "Rule-based face detection in frontal views," Proceedings of the International Conference on Acoustics, Speech, and Signal Processing, vol. 4, pp. 2537-2540, 1997.

[10] M. Lades, J. C. Vorbruggen, J. Buhmann, J. Lange, C. von de Malsburg, R. P. Wurtz, and W. Konen, "Distortion invariant object recognition in the dynamic link architecture," IEEE Transactions on Computers, vol. 42, pp. 300-311, 1993.

[11] R. Lienhart, A. Kuranov, and V. Pisarevsky "Empirical analysis of detection cascades of boosted classifiers for rapid object detection," Proceedings of the DAGM Pattern Recognition Symposium, pp. 297-304, 2003.

[12] C. Liu and H. Wechsler, "Gabor feature based classification using the enhanced fisher linear discriminant model for facce recognition," IEEE Transactions on Image Processing, vol. 11, no. 4, pp. 467-476, 2002.

[13] X. Liu, "A computational framework for real-time scene interpretation," in Proceedings of Applied Imagery Pattern Recognition Workshop, 2004.

[14] X. Liu and L. Cheng, "Independent spectral representation of images for recognition," Journal of the Optical Society of America, A, vol. 20, no. 7, pp. 1271-1282, 2003.

[15] X. Liu and A. Srivastava, "Stochastic search for the optimal linear representations of images on spaces with orthogonality constraints," in Proceedings of the International 
Workshop on Energy Minimization Methods in Computer Vision and Patter Recognition, pp. 3-20, 2003.

[16] X. Liu, A. Srivastava, and D. Wang, "Intrinsic generalization analysis of low dimensional representations," Neural Networks, vol. 16, no. 5/6, pp. 537-545, 2003.

[17] X. Liu and D. Wang, "A spectral histogram model for texton modeling and texture discrimination," Vision Research, vol. 42, pp. 2617-2634, 2002.

[18] X. Liu and D. Wang, "Texture classification using spectral histograms," IEEE Transactions on Image Processing, vol. 12, no. 6, pp. 661-670, 2003.

[19] B. Moghaddam and A. Pentland, "Probabilistic visual learning for object recognition," IEEE Transactions on Pattern Analysis and Machine Intelligence, vol. 19, no. 7, pp. 696-710, 1997.

[20] E. Osuna, R. Freund, and F. Girosi, "Training support vector machines: an application to face detection," in Proceedings of the IEEE Conference on Computer Vision and Patter Recognition, 1997, pp. 130-136.

[21] H. Rowley, S. Baluja, and T. Kanade, "Neural network-based face detection," IEEE Transactions on Pattern Analysis and Machine Intelligence, vol. 20, no. 1, pp. 23 - 38, 1998.

[22] H. Schneiderman, "A statistical approach to 3d object detection applied to faces and cars," Ph.D. dissertation, Carnegie Mellon University, 2000. 
[23] A. Srivastava and X. Liu, "Statistical hypothesis pruning for identifying faces from infrared images," Journal of Image and Vision Computing, vol. 21, no. 7, pp. 651-660, 2003.

[24] A. Srivastava, X. Liu, and U. Grenander, "Universal analytical forms for modeling image probabilities," IEEE Transactions on Pattern Analysis and Machine Intelligence, vol. 24, no. 9, pp. 1200-1214, 2002.

[25] K.-K. Sung and T. Poggio, "Example-based learning for view-based human face detection," IEEE Transaction on Pattern Analysis and Machine Intelligence, vol. 20, no. 1, pp. 39-51, 1998.

[26] V. Vapnik, The nature of statistical learning theory. New York: Springer-Verlag, 1995.

[27] P. Viola and M. J. Jones, "Robust real-time face detection," International Journal of Computer Vision, vol. 57, no. 2, pp. 137-154, 2004.

[28] C. A. Waring, "An exploration of the spectral histogram representation for face detection," Masters Thesis, Department of Computer Science, Florida State University, July, 2003.

[29] G. Wall, X. Liu, F. Iqbal, and S. Foo, "Real-time texture classification using FPGA," in Proceedings of Applied Imagery Pattern Recognition Workshop, 2004.

[30] G. Winkler, Image Analysis, Random Fields and Dynamic Monte Carlo Methods: A Mathematical Introduction, 2nd ed. Berlin: Springer, 2003. 
[31] M. Yang, N. Ahuja, and D. Kriegman, "Mixture of linear subspaces for face detection," in Proceedings of the International Conference on Automatic Face and Gesture Recognition, 2000, pp. 70-76.

[32] M. Yang, D. Kriegman, and N. Ahuja, "Detecting faces in images: a survey," IEEE Transactions on Pattern Analysis and Machine Intelligence, vol. 24, no. 1, pp. 34-58, 2002.

[33] M. Yang, N. Roth, and N. Ahuja, "A snow-based face detector," Advances in Neural Information Processing Systems, vol. 12, pp. 855-861, 2000.

[34] K. Yow and R. Cipolla, "Feature-based human face detection," Image and Vision Computing, vol. 15, no. 9, pp. 713-735, 1997.

[35] A. Yuille, D. Cohen, and P. Hallinan, "Feature extraction from faces using deformable templates," in Proceedings of the IEEE Conference on Computer Vision and Pattern Recognition, 1989, pp. '104-109.

[36] S. Zhu, X. Liu, and Y. Wu, "Exploring texture ensembles by efficient markov chain monte carlo," IEEE Transactions on Pattern Recognition and Machine Intelligence, vol. 22 , no. 6 , pp. $554-569,2000$.

[37] S. Zhu, Y. Wu, and D. Mumford, "Minmax entropy principle and its application to texture modeling," Neural Computation, vol. 9, pp. 1627-1660, 1997. 


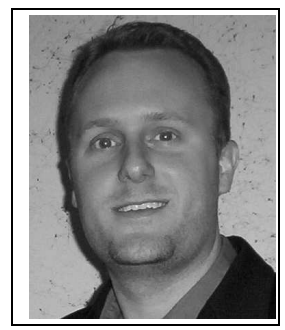

Christopher A. Waring received his B.S. in Pure Mathematics and Computer Science in 2002 and his M.S. in Computer Science in 2003 all from the Florida State University. His research interests include computer vision, pattern recognition, and their applications.

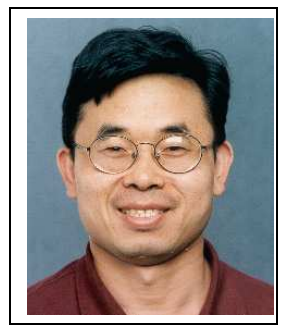

Xiuwen Liu received the B.Eng. degree in Computer Science in 1989 from Tsinghua University, Beijing, China, the M.S. degrees in Geodetic Science and Surveying in 1995 and Computer and Information Science in 1996, and Ph.D. in Computer and Information Science in 1999 from the Ohio State University, Columbus, OH. From August 1989 to February 1993, he was with Department of Computer Science and Technology at Tsinghua University. Since 2000, he has been with the Department of Computer Science of the Florida State University, Tallahassee, Florida. His current research interests include low dimensional representations of images, statistical pattern recognition, manifold-based optimization and inference algorithms, computational modeling of visual perception and inference, and real-time vision algorithms and implementations using FPGAs. 